\title{
Improved Version of Cancer Evo-Dev, a Novel Scientific Hypothesis Derived From Advances in Hepatitis B Virus (HBV)-Induced Hepatocarcinogenesis
}

\author{
Guangwen Cao* \\ Department of Epidemiology, Second Military Medical University, PR China \\ *Corresponding author: Guangwen Cao, Department of Epidemiology, Second Military Medical University, 800 Xiang-yin Rd., Shanghai 200433, PR \\ China
}

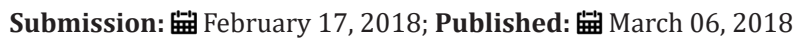

\begin{abstract}
A novel scientific hypothesis Cancer Evo-Dev is presented based on hepatitis B virus (HBV)-induced carcinogenesis. The framework of improved version covers following aspects. Interaction of genetic predispositions and environmental insults contribute to non-resolving inflammation. Inflammatory factors promote somatic mutations in the viral or human genomes via inducing imbalance between the mutagenic force and mutationrepairing force. The inflammatory microenvironment selects mutated viruses and the virus-altered host cells, and also drives retro-differentiation of the mutated host cells into cancer stem-like cells. Cancer stem-like cells preferentially utilize glycolysis for energy production to survive and also for generating the raw material to proliferate themselves in the hostile microenvironment. Key molecules involved in this evolutionary-developmental process of Cancer Evo-Dev can be applied for the prediction, prophylaxis, and targeted treatment of malignant diseases.
\end{abstract}

\section{Introduction}

Chronic but active inflammation, which is activated and maintained by stimulants such as infection and the interactions of stimulants with genetic predisposition, facilitates the occurrence and recurrence of cancers of various histotypes. Chronic inflammation, apparent or unapparent, is indispensible for the development of most malignancies, which has been clarified in hepatitis B virus (HBV)-induced hepatocellular carcinoma (HCC). Based on our previous work and the advances of researches on HBV-induced HCC and other inflammation-associated cancers, we presented the framework of a novel cancer theory termed Cancer Evolution and Development (Cancer Evo-Dev) [1-3]. Actually, Cancer Evo-Dev can be applied in cancers of many histotypes.

The major components of this theory are listed as follows.

I. Interaction of genetic predispositions of key immune- or inflammatory molecules and environmental insults including chronic HBV infection contributes to imbalance of immunity that is essential to arouse and maintain inflammation. We have found that the genetic polymorphisms of signal transducers and activators of

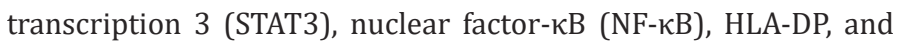
HLA-DQ are significantly associated with the generation of the endstage liver diseases-associated HBV mutations, the persistence of HBV infection, and increased risks of developing liver cirrhosis and HCC in chronic HBV-infected subjects [4-7].
II. Dysregulation of inflammatory cytokines including transforming growth factor $\beta 1$ (TGF- $\beta 1$ ) and interleukin- $1 \beta$ (IL-1 $\beta$ ) as well as proinflammatory signalling pathways including NF- $\mathrm{BB}$ and STAT3-signalling molecules such as tumor necrosis factor- $\alpha$ (TNF- $\alpha$ ) and IL-6 in immunosuppressive inflammatory environment promote somatic mutations in the human genomes or viral genome via inducing imbalances between the mutagenic forces including the activation-induced cytidine deaminase/apolipoprotein B mRNAediting enzyme catalytic subunit 3 members (AID/APOBEC3s), a family of cytidine deaminases and the mutation-repairing forces including uracil-DNA glycosylases (UNG), thus promoting somatic mutations in human genome and mutations in the HBV genome [8,9]. In malignancies of most histotypes, AID/APOBEC3s-driven somatic mutations alter a small number of key genes in cellular signalling pathways to incur a growth advantage of mutated cells. These somatic mutations are driver mutations. All of the known driver genes containing driver mutation(s) can be classified into 12 pathways of the 3 major functions: cell fate, cell survival, and genome maintenance. Cell fate function contains "NOTCH", "Hedgehog", "APC", "chromatin modification", and "transcriptional regulation" pathways. Cell survival contains "cell cycle/apoptosis", "RAS", "phosphoinositide 3- kinase", "STAT", "mitogen-activated protein kinase", and "TGF- $\beta$ ". Genome maintenance is governed by "DNA damage control" $[10,11]$. The mutated viruses should experience the same selection and adaptation processes [8]. AID/ 
APOBEC3s not only promote somatic mutation and viral mutation but also alter epigenetic modification of some genes by deaminating 5-methylcytosine or 5-hydroxymethylcytosine directly in concert with base-excision repair to exchange cytosine, thus promoting gene demethylation and removing epigenetic memory to stabilize the pluripotent state in embryonic stem cells. By this way, AID/ APOBEC3s promote epithelial-to-mesenchymal transition (EMT), a process that is critical in embryonic development and cancer metastasis [12]. Thus, AID/APOBEC3s bridge non-resolving inflammation and the occurrence and recurrence of cancers.

III. Most of the mutated cells and viruses are cleaned up in the hostile microenvironment. Only a small proportion of the mutated cells whose somatic mutations change their survival signalling adapt to the hostile microenvironment, retro-differentiate via promoting EMT by AID/APOBEC3s, and reversely develop into the so-called tumor-initiating cells or cancer stem cells. During the development from the embryo to adult, most genes expressed at the embryonic stage are going to be silenced after birth. Some fetal proteins such as $\alpha$-fetoprotein (AFP) or carcinoembryonic antigen (CEA) can re-express temporarily if some tissues like liver or testis quickly are injured and repaired. Interestingly, when some cancers develop, these fetal proteins can re-express persistently and serve as the biomarkers of cancers in the circulations of patients. Thus, carcinogenesis represents a reverse-developmental process, namely, transformation from differentiated cells into undifferentiated cells. The oncofetal protein AFP and CEA serve as diagnostic biomarkers of HCC and other gastrointestinal malignancies, respectively. Another oncofetal protein, SALL4, is a transcriptional factor that regulates developmental events, embryonic stem cell properties, and cancers. The expression of SALL4 is high in human fetal liver and silenced in the adult liver, but it has been proven to be re-expressed in some HCC. The re-expression of SALL4 in HCC subsets represents the "stemness" that confers the invasion and unfavourable prognosis [13]. Embryonic or stem-like gene signatures expressed in the tumors of diverse histotypes are robustly related to EMT and increased self-renewal and aggressiveness of cancer cells as well as poor postoperative prognosis [14]. During cancer invasion via EMT, epithelial cancer cells acquire "stemness," including selfrenewal and anti-apoptotic capacities. Within the tumor masses, only a small proportion of tumor cells with the "stemness" feature become the major malignant cell subpopulations in the different branches of primary and metastatic masses, thus forming tumor heterogenicity. These tumor subpopulations should be cancer stem cells of distinct potentials, contributing to the metastatic nature and chemo-resistance. Thus, retro-differentiation and reversedevelopment are the major characteristics of cancer development.

IV. Under the cultivation of tumor-promoting factors such as POSTN from tumor-infiltrating neutrophils, tumor-infiltrating fibroblasts, and tumor-infiltrating macrophages (M2 type), tumorinitiating cells acquire the "stemness". Cancer-stem cells survive under the selection procedure from distinct microenvironments and acquire capabilities of distant metastasis and drug-resistance [15].
V. Cancer cells preferentially utilize glycolysis for energy production, even in the presence of oxygen, to support rapid growth of cancer cells, a phenomenon known as the Warburg effect. The Warburg effect in M2 macrophages promotes the formation of vascular network, augments extravasation of tumour cells, and induces a higher level of EMT within the tumors [16]. The Warburg effect provides not only essential energy for cell survival but also the raw material for DNA synthesis in the fast proliferating cells.

Thus, the development of malignant disease is characterized by an evolutionary process of "mutation-selection-adaptation". The framework of improved Cancer Evo-Dev can be verified in other cancers such as cancers of the breast, cervix, head \& neck, colon \& rectum, ovary, stomach, biliary tract and lung \& bronchi $[9,17]$. Key molecules involved in the critical steps of the evolutionarydevelopmental process can be applied for the prediction of occurrence and postoperative recurrence as well as specific prophylaxis of malignant diseases. In addition, the key molecules in the functional signalling pathways that contribute to the stemness and promote cancer evolutionary-developmental process can serve as specific therapeutic targets for the inhibitors, thus providing a specific therapeutic strategy for a given advanced malignant disease. Thus, Cancer Evo-Dev has the following major functions in cancer prevention and control. The first, it can be applied to identify which precancerous lesion is more likely to develop cancers and to reduce the occurrence of cancers via removing or treating the dangerous precancerous lesions. The second, it can be applied to test what kinds of prophylactic and/or therapeutic options can reduce the incidence of malignancy and delay the postoperative occurrence. The third, it helps in developing individualized targeted therapy to block the key signalling pathways specifically and terminate the evolution and development of malignancies.

\section{Funding}

Supported by the National 973 Program (2015CB554000) and the National Natural Scientific Foundation of China (91129301).

\section{References}

1. Cao GW (2017) Cancer Evo-Dev, a novel hypothesis derived from studies on hepatitis B virus-induced carcinogenesis. Hepatoma Res 3: 241-259.

2. Liu WB, Wu JF, Du Y, Cao GW (2016) Cancer Evolution-Development: experience of hepatitis B virus-induced hepatocarcinogenesis. Curr Oncol 23(1): e49-e56.

3. Ji X, Zhang Q Du Y, Liu W, Li Z, et al. (2014) Somatic mutations, viral integration and epigenetic modification in the evolution of hepatitis $B$ virus-induced hepatocellular carcinoma. Curr Genomics 15(6): 469-480.

4. Zhang Q Yin J, Zhang Y, Deng Y, Ji X, et al. (2013) HLA-DP polymorphisms affect the outcomes of chronic hepatitis B virus infections, possibly through interacting with viral mutations. J Virol 87(22):12176-12186.

5. Ji X, Zhang Q Li B, Du Y, Yin J, et al. (2014) Impacts of human leukocyte antigen DQ genetic polymorphisms and their interactions with hepatitis $B$ virus mutations on the risks of viral persistence, liver cirrhosis, and hepatocellular carcinoma. Infect Genet Evol 28: 201-209.

6. Xie J, Zhang Y, Zhang Q Han Y, Yin J, et al. (2013) Interaction of signal transducer and activator of transcription 3 polymorphisms with hepatitis B virus mutations in hepatocellular carcinoma. Hepatology 


\section{7(6): 2369-2377.}

7. Zhang Q Ji XW, Hou XM, Lu FM, Du Y, et al. (2014) Effect of functional nuclear factor-kappaB genetic polymorphisms on hepatitis B virus persistence and their interactions with viral mutations on the risk of hepatocellular carcinoma. Ann Oncol 25(12): 2413-2419.

8. Deng Y, Du Y, Zhang Q, Han X, Cao G (2014) Human cytidine deaminases facilitate hepatitis $B$ virus evolution and link inflammation and hepatocellular carcinoma. Cancer Lett 343(2):161-171.

9. Kuong KJ, Loeb LA (2013) APOBEC3B mutagenesis in cancer. Nat Genet 45(9): 964-965.

10. Vogelstein B, Papadopoulos N, Velculescu VE, Zhou S, Diaz LA, et al. (2013) Cancer genome landscapes. Science 339(6127): 1546-1558.

11. Hou X, Yang F, Liu W, Fu Z, Chen L, et al. (2015) Signaling pathways that facilitate chronic inflammation-induced carcinogenesis. J Cell Signal 1: 104.

12. Muñoz DP, Lee EL, Takayama S, Coppé JP, Heo SJ, et al. (2013) Activationinduced cytidine deaminase (AID) is necessary for the epithelialmesenchymal transition in mammary epithelial cells. Proc Natl Acad Sci

\section{U S A 110(32): E2977-E2986.}

13. Yong KJ, Gao C, Lim JS, Yan B, Yang H, et al. (2013) Oncofetal gene SALL4 in aggressive hepatocellular carcinoma. N Engl J Med 368(24): 22662276.

14. Carro MS, Lim WK, Alvarez MJ, Bollo RJ, Zhao X, et al. (2010) The transcriptional network for mesenchymal transformation of brain tumours. Nature 463(7279): 318-325.

15. Xu X, Chang W, Yuan J, Han X, Tan X, et al. (2016) Periostin expression in intra-tumoral stromal cells is prognostic and predictive for colorectal carcinoma via creating a cancer-supportive niche. Oncotarget 7(1): 798813.

16. Penny HL, Sieow JL, Adriani G, Yeap WH, See Chi Ee P, et al. (2016) Warburg metabolism in tumor-conditioned macrophages promotes metastasis in human pancreatic ductal adenocarcinoma. Oncoimmunology 5(8): e1191731.

17. Nakumara H, Arai Y, Totoki Y, Shirota T, Elzawahry A, et al. (2015) Genomic spectra of biliary tract cancer. Nat Genet 47(9): 1003-10010.

\section{Your subsequent submission with Crimson Publishers} will attain the below benefits

Creative Commons Attribution 4.0

International License

For possible submissions Click Here 